\title{
PSYCHE
}

\begin{tabular}{lll}
\hline VOL. XXII & AUGUST, 1915 & No. 4
\end{tabular}

\section{A PRELIMINARY LIST OF THE ANTHOPHILOUS COLEOPTERA OF NEW ENGLAND.}

\author{
By John H. Lovell, \\ Waldoboro, Maine.
}

The following list of the flower-visiting beetles of New England is based on observations extending over more than ten years. No records are given of visits that were known to be accidental, or of captures that were made by sweeping. The writer has worked only at Waldoboro, Maine; all other records whether in Maine or Massachusetts should be credited to Mr. C. A. Frost of Framingham, Mass. To avoid needless repetition the following abbreviations for localities are employed: F. for Framingham, S. for Sherborn, and H. for Hopkinton, Mass.; and W. for Waldoboro, Maine. In the determination of the more difficult species the authors desire to acknowledge the assistance of Mr. E. A. Schwarz, Col. T. L. Casey, Mr. Charles Dury and Mr. W. S. Fisher; the larger part of the material taken at Waldoboro, Maine, was some years ago kindly identified by Professor H. F. Wickham. Where only the generic names Cornus and Viburnum appear in the list the species may usually be understood to be $C$. alternifolia and V. nudum.

$$
\text { Carabide. }
$$

Lebia atriventris Say. Solidago, S.

L. fuscata Dej. Prunus virginiana, S.

L. furcata Lec. Solidago, S.

L. ornata Say. Solidago, Cornus, Eupatorium perfoliatum, F. Amelanchier canadensis, S. Viburnum, H. Solidago rugosa, W.

L. pectita Horn. Solidago, F.

L. pumila Dej. Solidago, $\mathbf{H}$.

L. viridis Say. Solidago, Spiræa salicifolia, Amelanchier canadensis, Viburnum, F. Prunus virginiana, $H$. Cornus alternifolia, W.

L. viridis var. mosta Lec. Solidago, F.

L. viridipennis Dej. Solidago, S. 
Phalacridee.

Olibrus pallipes Say. Solidago, Natick, Mass.

Phalacrus politus Say. Cornus, S.

\section{Staphylinide.}

Anthobium convexum Fauv. Viburnum, Pyrus arbutifolia, S. Acer spicatum, W. In Stuben County, Ind., this species is common on the flowers of Sambucus racemosa and Viburnum prunifolium. ${ }^{1}$

A. pothos Mann. Pyrus arbutifolia, F. Clintonia borealis, W.

Arpedium tenue Lec. Viburnum, Monmouth, Me.

Homalium rufipes Fauv. Viburnum, S.

Placusa tacomœ Casey. Viburnum, Monmouth, Me.

\section{Coccinellide.}

Adalia bipunctata L. Pyrus communis, H. Viburnum, S.

A. humeralis Say. Salix discolor, Asclepias, F.

Anisosticta strigata Thun. On flowers of an early grass.

Brachyacantha ursina Fab. Cornus, Southboro, Mass. Chrysanthemum Leucanthemum, W.

Coccinella sanguinea L. Cornus, Southboro, Mass. Solidago, S.

C. trifasciata L. Amelanchier canadensis, Viburnum, S. Ribes prostratum, W.

C. transversoguttata Fab. Asclepias, F.

Hippodamia glacialis Fab. Asclepias, F.

H. convergens Guen. Asclepias, Solidago, F.

H. 13-punctata L. Solidago, F. Taraxicum officinale, W.

H. parenthesis Say. Spiræa salicifolia, Daucus Carota, F.

Hyperaspis undulata Say. Daucus Carota, S.

H. octavia Casey. Salix, Natick, Mass.

Megilla maculata DeG. Eupatorium perfoliatum, Solidago, Aster, Daucus Carota, Brassica, S.

Psyllobora 20-maculata Say. Solidago, F.

Scymnus americanus Muls. Amelanchier canadensis, S.

S. indutus Casey. Prunus virginiana, S.

\section{Cryptophagide.}

Antherophagus ochraceus Melsh. Spiræa salicifolia, Monmouth, Me. Rosa humilis, W.

Telmatophilus (Loberus) impressus Lec. Viburnum, F.

Tomarus pulchellus Lec. Amelanchier canadensis, S.

\section{Erotylide.}

Acropteroxys gracilis Newm. Rosa humilis, F.

\footnotetext{
1 “The Coleoptera in Indiana," p. 481.
} 
Dermestide.

Anthrenus castanee Melsh. Aralia hispida, W.

A. musœorum L. Spiræa salicifolia, Soldiago, Eupatorium perfoliatum, Viburnum,

Cornus, S. Achillea Millefolium, Paris, Me. Thalictrum polygamum, Chrysanthemum Leucanthemum, Monmouth, Me.

A. scrophularice L. Pyrus arbutifolia, Cornus, F. Prunus virginiana, H. Spiræa salicifolia, Viburnum, Natick, Mass.

A. verbasci Oliv. Viburnum, Natick, Mass.

Attagenus pellio L. Amelanchier canadensis, Pyrus communis, $\mathrm{H}$.

A. piceus Oliv. Cornus, Viburnum, Rubus, F.

Eyturus unicolor Say. Prunus virginiana, H. Rubus idæus, Monmouth, Me. Anemone quinquefolia, W.

Cryptorhopalum hœmorrhoidale Lec. Spiræa salicifolia, F. Viburnum, Monmouth, Me.

C. picicorne Lec. Fragaria virginiana, F. Cornus, Viburnum, Pyrus arbutifolia

S. Prunus virginiana, Solidago, $\mathrm{H}$.

C. triste Lec. Solidago, S.

\section{Nitidulide.}

Carpophilus brachypterus Say. Pyrus arbutifolia, Prunus virginiana, Cornus, F. Amelanchier canadensis, Prunus pennsylvanica, Pyrus communis, H. Viburnum Lentago, W.

Cercus abdominalis Er. Amelanchier canadensis, Pyrus communis, H. Spiræa salicifolia, Pyrus arbutifolia, Viburnum, S. Sambucus canadensis, Monmouth, Me.

Conotelus obscurus Er. Convolvulus, Sambucus canadensis, F. Monmouth, Me. Cornus, Southboro, Mass.

Epurca sp. Viburnum, Monmouth, Me.

Colastus truncatus Rand. Viburnum Lentago, W.

\section{LATHRIDIIDA.}

Melanophthalma distinguenda Com. Amelanchier canadensis, F.

Dascyllide.

Cyphon collaris Guer. Prunus virginiana, S.

C. obscurus Guer. Viburnum, Natick, Mass.

C. padi $\mathbf{L}$. Pyrus arbutifolia, $\mathrm{H}$.

C. variabilis Thumb. Salix discolor, F. Amelanchier canadensis, Prunus virginiana, S. Salix discolor, W.

\section{ELATERIDA.}

Agriotes stabilis Lec. Rubus idæus, Monmouth, Me. Viburnum nudum, W. A. oblongicollis Melsh. Prunus virginiana, Viburnum, S.

A. fucosus Lec. Viburnum nudum, W.

A. sp. Prunus virginiana, W.

Asaphes decaloratus Say. Chrysanthemum Leucanthemum, W. 
Corymbites hamatus Say. Acer spicatum, W.

C. inflatus Say. Prunus virginiana, $\mathbf{H}$.

C. hieroglyphicus Say. Prunus virginiana, H. Acer spicatum, W.

C. medianus Germ. Prunus virginiana, $\mathrm{H}$.

C. metallicus Payk. Cornus stolonifera, W.

C. tarsalis Melsh. Amelanchier canadensis, S. Prunus virginiana, H. Salix, Viburnum, Natick, Mass.

C. tessellatus $\mathrm{L}$. Rubus allegheniensis, $\mathrm{S}$.

C. triundulatus Rand. Prunus virginiana, Amelanchier canadensis, S.

C. vernalis Hentz. Prunus serotina, Wales, Me.

Cardiophorus sp. Cornus canadensis, W.

Dolopius lateralis Esch. Geranium maculatum, Prunus virginiana, Viburnum, S.

Viburnum alnifolium, W.

Ela'er collaris Say. Viburnum, Natick, Mass. Aralia hispida, W.

E. militaris Harr. Viburnum, S.

E. miniipennis Lec. Pyrus arbutifolia, H. Viburnum, F. Spiræa salicifolia, W.

E. nigricans Germ. Amelanchier canadensis, S.

E. nigricollis Hbst. Amelanchier canadensis, S.

E. pedalis Germ. Amelanchier canadensis, F. Cornus, S. Pyrus arbutifolia, Wales, Me.

E. rubricus Say. Amelanchier canadensis, Prunus virginiana, Viburnum, Rubus, F. Viburnum alnifolium, W.

Megapenthes rogersii Horn. Viburnum alnifolium, W.

Melanotus americanus Hbst. Cornus, F.

M. communis Gyll. Viburnum, Natick, Mass.

M. leonardi Lec. Rubus idæus, Paris, Me.

M. sagittarius Lec. Viburnum, Natick, Mass.

M. trapezoideus Lec. Amelanchier canadensis, S.

Limonius confusus Lec. Prunus virginiana, Viburnum, S.

L. basilaris Say. Crataegus, H. Viburnum, Natick, Mass. Cornus, F.

L. griseus Beauv. Cornus, S.

L. nitidulus Horn. Viburnum, S.

L. plebejus Say. Pyrus arbutifolia, Prunus virginiana, H. Cornus, F. Viburnum, Natick, Mass.

L. stigma Hbst. Prunus virginiana, H.

Estodes tenuicollis Rand. Chrysanthemum Leucanthemum, W.

Oxygonus obesus Say. Viburnum, Monmouth, Me.

Sericus silaceus Say. Viburnum, Monmouth, Me.

Sericosomus incongruus Lec. Viburnum dentatum, W.

\section{Buprestide.}

Acmæodera tubulus Fab. Geranium maculatum, Viburnum, Rubus idæus, Achillea Millefolium, S. Prunus virginiana, Amelanchier canadensis, Cornns florida, $\mathbf{H}$. 
LAMPYRIDA.

Chauliognathus pennsylvanicus DeG. Solidago, F., W., and Monmouth, Me.

Ellychnia corrusca L. Amelanchier canadensis, S. Cratægus, H. Cornus canadensis, $\mathbf{W}$.

Lucidota atra Fab. Iris versicolor, W.

Podabrus nothoides Lec. Acer spicatum, W.

$\boldsymbol{P}$. tricostatus Say. Viburnum, Natick, Mass.

$P$. rugulosus Lec. Cornus, F.

Pyractomena angulata Say. Aralia hispida, W.

Pyropyga decipiens Harr. Cornus, Southboro, Mass. Aralia hispida, W.

$P$. nigricans Say. Cornus, Southboro, Mass.

Telephorus bilineatus Say. Prunus virginiana, H.

T. carolinus Fab. Cornus, Natick, Mass.

T. excaratus Lec. Rubus, Viburnum, S.

T. fraxini Say. Brassica campestris, Natick, Mass. Cornus canadensis, Aralia hispida, W.

T. lineola Fab. Viburnum, Prunus, S.

T. rectus Mesh: Cornus, Southboro, Mass.

T. rotundicollis Say. Viburnum, S.

T. scitulus Say. Epilobium angustifolium, W.

T. tuberculata Lec. Brassica campestris, Natick, Mass.

\section{MaLACHIID}

Attalus terminalis Er. Ceanothus americanus, Prunus virginiana, S. Achillea Millefolium, Paris, Me. Rhus typhina, W.

A. scincetus Say. Cornus florida, Prunus virginiana, H. Viburnum, S.

Collops nigriceps Say. Prunus virginiana, $\mathrm{H}$.

Malachius oneus L. Viburnum, Natick, Mass.

Pseudobøus oblitus Lec. Prunus virginiana, $\mathrm{H}$.

\section{Clerida.}

Trichodes nuttalli Kirby. Spiræa salicifolia, Solidago, Monmouth, Me. Chrysanthemum Leucanthemum, W.

Laricobius erichsoni Rosen. Salix, S.

\section{SCARABAEIDE.}

A phodius fimetarius L. Spiræa salicifolia, W.

Amphicoma vulpina Hentz. Achillea Millefolium, F. Spiræa, Monmouth, Me.

Euphoria fulgida Fab. Prunus virginiana, H. and Monmouth, Me.

Hoplia trifasciata Say. Amelanchier canadensis, Prunus virginiana, Viburnum, Cornus, F. Viburnum dentatum, Vaccinium vacillans, W.

H. tristis Melsh. Salix discolor, Amelanchier canadensis, F. Prunus virginiana, S. Macrodactylus subspinosus Fab. Spiræa salicifolia, Viburnum, Cornus, F. Achillea Millefolium, Sambucus canadensis, Southboro, Mass. Viburnum dentatum, W. 
Serica tristis Lec. Rubus allegheniensis, Wales, Me.

Trichius affinis Gory. Spiræa salicifolia, Rubus, Viburnum, Cornus, F. Viburnum dentatum, W.

T. piger Fab. Spiræa salicifolia, Cornus, F. Viburnum, S.

\section{Cerambycide.}

Acmcops bivittata Say. Pyrus americana, Mt. Washington, N. H.

A. pratensis Laich. Achillea Millefolium, Paris, Me.

Callimoxys sanguinicollis Oliv. Prunus virginiana, Viburnum acerifolium, $\mathbf{H}$.

Cyllene robinioe Forst. Solidago, Brookline, Mass.

Clytanthus ruricola Oliv. Ceanothus americanus, F. Epilobium angustifolium, Eupatorium perfoliatum, W.

Cyrtophorus verrucosus Oliv. Prunus virginiana, H. Viburnum alnifolium, W.

Euderces picipes Fab. Ceanothus americanus, Spiræa salicifolia, Viburnum, F. Rhus vernix, Southboro, Mass.

Elytroleptus floridanus Lec. Occasionally taken on the flowers of the oak (Quercus), Natick, Mass.

Gaurotes cyanipennis Say. Viburnum, Cornus, F. Prunus virginiana, H.

Microclytus frosti Casey. Viburnum, Paris, Me.

M. gazellula Hald. Viburnum alnifolium, W.

Molorchus bimaculatus Say. Cornus florida, Prunus virginiana, Viburnum, $\mathbf{H}$.

Viburnum nudum, W.

Pachyta monticola Rand. Spiræa salicifolia, Paris, Me. Viburnum nudum, W.

Leptura bifloris Newm. Spiræa salicifolia, Monmouth, Me.

L. capitata Newm. Viburnum, Natick, Mass.

L. canadensis Fab. Spiræa salicifolia, Wales, Me. Solidago serotina, W.

L. circumdata Oliv. Ceanothus americanus, Prunus virginiana, Viburnum, Cornus, $\mathrm{S}$.

L. cordifera Oliv. Ceanothus americanus, Cornus, Solidago, S.

L. chrysocoma Kirby. Viburnum dentatum, Aralia hispida, W.

L. deleta Lec. Spiræa salicifolia, S.

L. lineola Say. Prunus virginiana, Spiræa salicifolia, Cornus florida, Zizia aureum, H. Cornus alternifolia, W.

L. mutabilis Newm. Ceanothus americanus, Prunus virginiana, S. Viburnum nudum, W.

L. hæmatites Newm. Cornus florida, Vibernum, H. Cornus alternifolia, S.

L. nitens Forst. Cornus alternifolia, S.

L. pedalis Lec. Pyrus americana, Mt. Washington, N. H.

L. pubera Say. Viburnum, S. Rubus idæus, Viburnum, Monmouth, Me.

L. proxima Say. Thalictrum polygamum, Viburnum, Monmouth, Me. Spiræa salicifolia, Rhus typhina, W.

L. octonotata Say. Viburnum, F.

L. subargentata Kirby. Cornus, Viburnum, S. Viburnum, Mt. Washington, N. H. Thalictrum polygamum, Monmouth, Me.

L. subhamata Rand. Spiræa salicifolia, Wales, Me.

L. rubrica Say. Spiræa salicifolia, S. 
L. vagans Oliv. Ceanothus americanus, Spiræa salicifolia, S. Aralia hispida, W.

L. vittata Germ. Spiræa salicifolia, Ceanothus americanus, S. Aralia hispida, Cornus alternifolia, W.

Strangalia acuminata Oliv. Rhus vernix, H. Spiræa salicifolia, S. Cornus, Viburnum, Southboro, Mass.

S. famelica Newm. Spiræa salicifolia, F.

S. luteicornis Fab. Spiræa salicifolia, S.

Saperda candida Fab. Solidago rugosa, W.

Typocerus velutinus Oliv. Eupatorium perfoliatum, Solidago, F. Viburnum, Asclepias, S. Solidago bicolor, W.

Spiræa salicifolia, Solidago, Thalictrum polygamum, Monmouth, Me.

Xylotrechus colonus Fab. Viburnum, Ashland, Mass.

\section{Chrysomelide.}

Calligrapha philadelphica L. Amelanchier canadensis, S.

Crepidodera helixenes $\mathrm{L}$. Prunus virginiana, $\mathrm{H}$.

Chrysodina globosa Say. Ceanothus americanus, F.

Chrysomela scalaris Lec. Cornus alternifolia, W.

Diabrotica atripennis Say. Solidago, $\mathrm{H}$.

D. 12-punctata Oliv. Solidago, Aster, F. Convolvulus sepium, W.

D. vittata Fab. Amelanchier canadensis, Prunus virginiana, Salix, Viburnum, Cornus, S. Solidago rugosa, W.

Diachus auratus Fab. Cornus, F. Achillea Millefolium, Paris, Me. Spiræa salicifolia, Diplopappus umbellatus, W.

Disonycha pennsylvanica Ill. Prunus virginiana, Amelanchier canadensis, Pyrus communis, $\mathbf{H}$.

Donacia piscatrix Lac. Nymphæa advena, W.

D. œqualis Say. Sagittaria latifolia, W.

D. rufa Say. Iris versicolor, W.

Galerucella nymphœœ L. Nymphæa advena, W.

Luperodes meraca Say. Viburnum, F.

Monachus ater Hald. Solidago, F.

Nodonota puncticollis Say. Spiræa salicifolia, Monmouth, Me. Rosa humilis, W. Edionychis limbalis Melsh. Amelanchier canadensis, S.

Orsodachna atra Ahr. Amelanchier canadensis, Salix, H. Six varieties on Viburnum, Paris, Me. Cornus alternifolia, W.

Pachybrachys subfasciatus Hald. Prunus virginiana, Cratægus, H.

Triachus cerinus Lec. Ceanothus americanus, S.

BRUCHIDE.

Bruchus calvus Horn. Spiræa salicifolia, Solidago, F.

B. obtectus Say. Amelanchier canadensis, S.

Cistelid

Cistela sericea Say. Spiræa salicifolia, Castanea dentata, F.

Isomira quadristriata Coup. Prunus virginiana, H. Cornus alternifolia, Acer spicatum, Rhus typhina, W. 


\section{MELANDRYide.}

Mycterus scaber Hald. Spiræa salicifolia, Ceanothus americanus, Solidago, Cornus, $\mathbf{F}$.

\section{CEDEMERID 2.}

Asclera ruficollis Say. Amelanchier canadensis, F. Prunus virginiana, H. Thalictrum polygamum, Monmouth, Me. Viburnum alnifolium, W.

\section{Cephaloönide.}

Cephaloön lepturides Newm. Viburnum, Milton, Mass. Cornus alternifolia, W.

\section{MORDELLIDA.}

Anaspis flavipennis Hald. Cornus florida, Prunus virginiana, H. Spiræa salicifolia, F. Thalictrum polygamum, Monmouth, Me.

A. rufa Say. Prunus virginiana, Viburnum, H. Thalictrum polygamum, Monmouth, Me. Viburnum dentatum, Aralia hispida, Polygonum cilinode, W.

Mordella melœna Germ. Spiræa salicifolia, Wales, Me.

M. marginata Melsh. Spiræa salicifolia, S. Viburnum dentatum, Aralia hispida, Rosa humilis, Rhus typhina, W.

M. scutellaris Fab. Prunus virginiana, Natick, Mass. Spiræa salicifolia, W.

Mordellistena aspersa Melsh. Viburnum, Natick, Mass.

M. biplagiata Helm. Spiræa salicifolia, Southboro, Mass. Viburnum nudum, W.

$M$. comata Lec. Spiræa salicifolia, Eupatorium purpureum, Natick, Mass.

$M$. intermixta Helm. Viburnum, S.

M. limbalis Melsh. Spiræa salicifolia, S.

M. unicolor Lec. Eupatorium purpureum, S.

\section{ANTHICIDE.}

Corphyra lugubris Say. Cornus alternifolia, Veratrum viride, Viburnum Lentago, W.

\section{Meloide.}

Epicauta pennsylvanica DeG. Aster, Solidago, S. Solidago lanceolata, W. Pomphopæa œnea Say. Amelanchier canadensis, $\mathrm{H}$.

$P$. sayi Lec. Ceanothus americanus, F. Prunus virginiana, H.

\section{RHIPIPHORIDE.}

Myodites fasciatus Say. Solidago, $\mathbf{F}$.

Rhipiphorus limbatus Fab. Solidago, S.

\section{Rhynchitidae.}

Rhynchites bicolor Fab. Rosa humilis, F. 


\section{Curculionide.}

Anthonomus corvulus Lec. Pyrus arbutifolia, Prunus virginiana, H. Cornus alternifolia, W.

A. moleculus Casey. Fragaria virginiana, F.

A. quadrigibbus Say. Prunus virginiana, F.

A. signatus Say. Rubus allegheniensis, $\mathrm{S}$.

Balaninus obtusus Blanch. Cornus, F.

Centrinus scutellum-album Say. Chrysanthemum Leucanthemum, Spiræa salicifolia, $\mathrm{S}$.

Conotrachelus nenuphar Hbst. Prunus virginiana, H. W.

Dorytomus brevicollis Lec. Salix discolor, S.

Gercus picumnus Hbst. Viburnum, Aster, F.

Listronotus appendiculatus Boh. Sagittaria latifolia, W.

Lixus concavus Say. Ranunculus, F.

Madarus undulatus Say. Solidago, F.

Mononychus vulpeculus Fab. Iris versicolor, F. W.

Nicentrus lineicollis Boh. Ceanothus americanus, Solidago, F.

Orchestes pallicornis Say. Amelanchier canadensis, S.

Piazorhinus scutellatus Say. Viburnum, F.

Pseudanthonomus cratogi Walsh. Amelanchier canadensis, Prunus virginiana, F. Rhinoncus pyrrhopus Boh. Rumex acetosella, F.

\section{PARANOMOPONE, A NEW GENUS OF PONERINE ANTS FROM QUEENSLAND. ${ }^{1}$}

\section{By William Morton Wheeler.}

Paranomopone gen. nov.

Worker. Rather small, monomorphic. Mandibles triangular, with toothed apical borders. Clypeus short, not produced in the middle, indistinctly marked off from the remainder of the head. This is also true of the triangular frontal area. Frontal carinæ distinctly dilated, further apart than the distance of each to the lateral border of the head, concealing the insertions of the antennæ, continued back about three-fourths the length of the head as the medial border of a deep scrobe which lies entirely dorsal to the eye. The ventral border of this scrobe, which is somewhat more than three times as long as broad, is also very sharp. Its depression is divided by a median longitudinal ridge which does not extend to the posterior end of the scrobe, so that the latter is incompletely separated into two compartments, one for the accommodation of the scape and one for the funiculus, when

\footnotetext{
${ }^{1}$ Contributions from the Entomological Laboratory of the Bussey Institution, Harvard University, No. 93.
} 

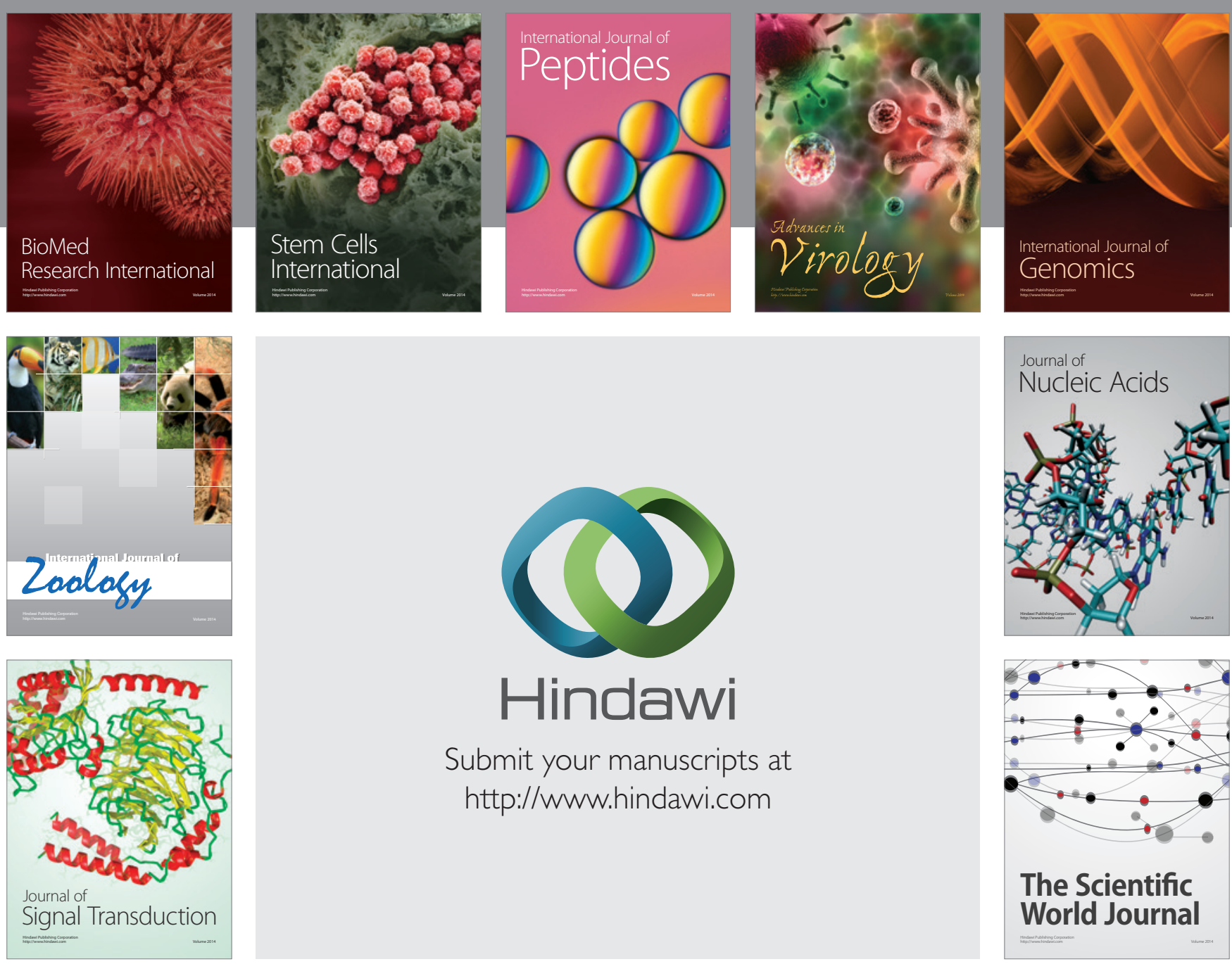

Submit your manuscripts at

http://www.hindawi.com
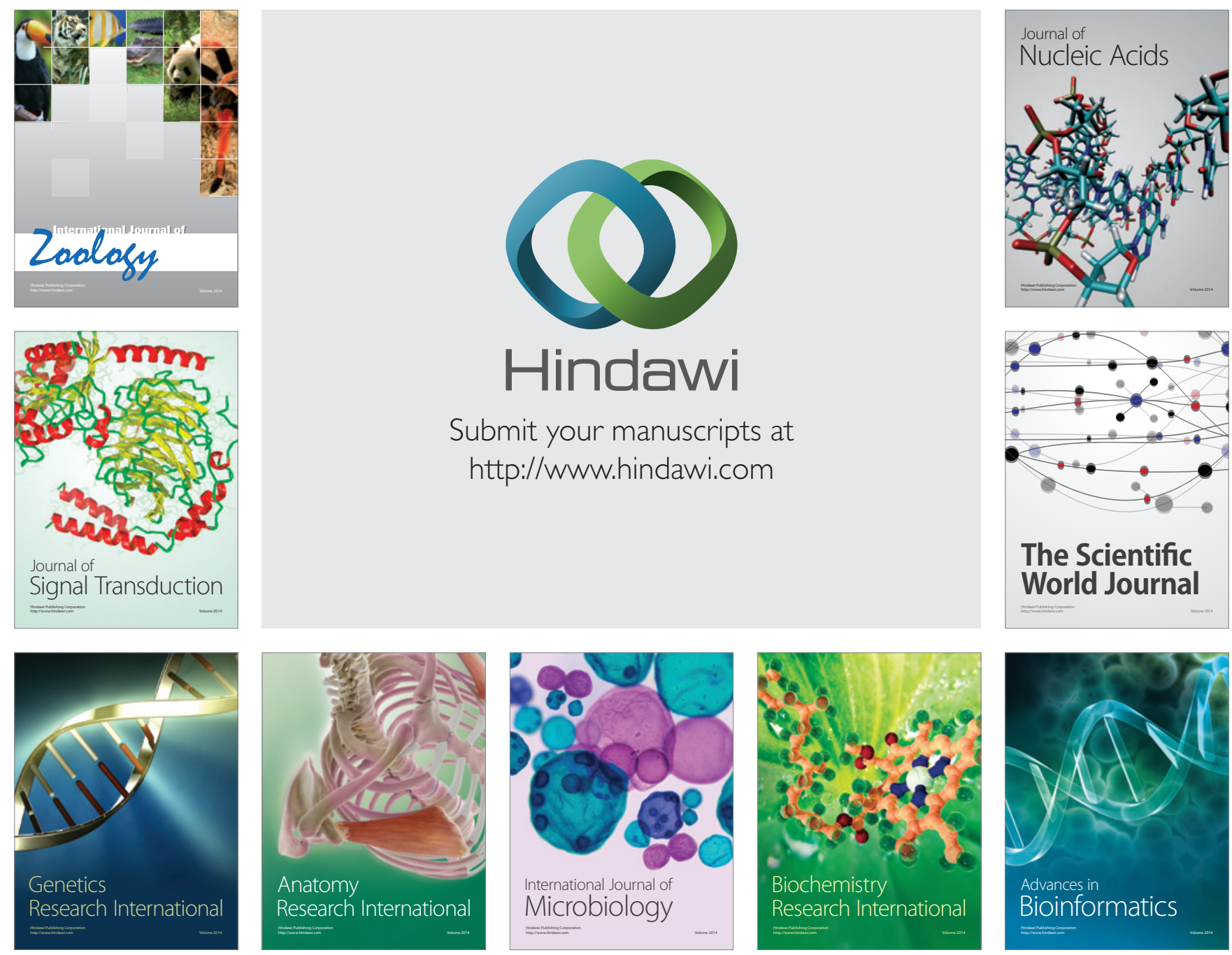

The Scientific World Journal
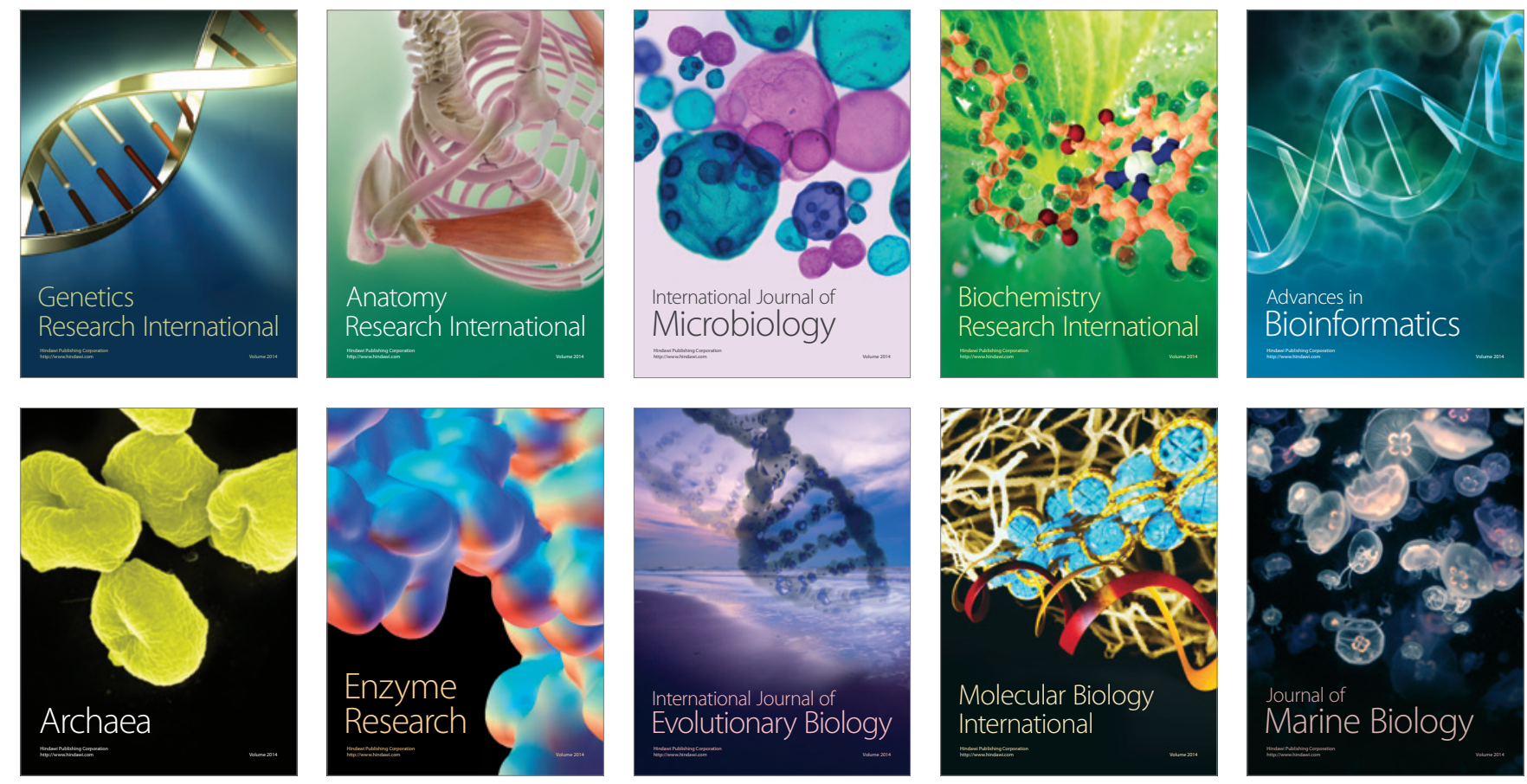